\title{
On Singular Dissipative Fourth-Order Differential Operator in Lim-4 Case
}

\author{
Ekin Ŭgurlu and Elgiz Bairamov \\ Department of Mathematics, Ankara University, Tandoğan, 06100 Ankara, Turkey \\ Correspondence should be addressed to Elgiz Bairamov; bairamov@science.ankara.edu.tr
}

Received 26 June 2013; Accepted 25 July 2013

Academic Editors: D. D. Hai and W. Shen

Copyright (C) 2013 E. Uğurlu and E. Bairamov. This is an open access article distributed under the Creative Commons Attribution License, which permits unrestricted use, distribution, and reproduction in any medium, provided the original work is properly cited.

A singular dissipative fourth-order differential operator in lim-4 case is considered. To investigate the spectral analysis of this operator, it is passed to the inverse operator with the help of Everitt's method. Finally, using Lidskiîs theorem, it is proved that the system of all eigen- and associated functions of this operator (also the boundary value problem) is complete.

\section{Introduction}

In 1910, Weyl showed that [1] the singular second-order differential operators can be fallen into two classes: operators in limit-circle case and operators in limit-point case. The operators in limit-circle case have the solutions that are all in square integrable space. However, in limit-point case, only one linearly independent solution can be in square integrable space. The development of this theory belongs to Titchmarsh [2]. After these fundamental works, second-order singular differential operators have been developed by many authors (e.g., see [3-6]).

Following the methods of Weyl and Titchmarsh, the theory for higher order equations and Hamiltonian systems was constructed in [7-19].

In [7], a regular self-adjoint fourth-order boundary value problem was investigated. Further Green's function and the resolvent operator were constructed. In $[8,13]$, such a construction was done for the singular self-adjoint fourth order boundary value problem. In $[9,10]$, higher-order differential equations whose coefficients are complex-valued were studied. In 1974, Walker [20] showed that an arbitrary order selfadjoint eigenvalue problem can be represented as an equivalent self-adjoint Hamiltonian systems. Further, the developments in the theory of singular self-adjoint Hamiltonian systems were given in [14-19].

On the other hand, an important class of the nonselfadjoint operators is the class of the dissipative operators [21].
It is known that all eigenvalues of the dissipative operators lie in the closed upper half-plane, but this analysis is so weak. There are some methods to complete the analysis of the dissipative operators. Some of them are Livšic's, Krein's, and Lidskiîs theorems and functional model [21,22]. These methods were used in the literature for the second-order differential operators (see [22-26]). In this paper a singular dissipative fourth order differential operator in lim- 4 case is investigated. In particular, using Lidskiîs theorem, it is shown that the system of all eigen- and associated functions is complete in $L^{2}(\Lambda)$.

\section{Preliminaries}

Let $L$ denote the linear nonself-adjoint operator in the Hilbert space $H$ with the domain $D(L)$. The element $y \in D(L), y \neq 0$, is called a root function of the operator $L$ corresponding to the eigenvalue $\lambda_{0}$, if all powers of $L$ are defined on this element and $\left(L-\lambda_{0} I\right)^{n} y=0$ for some $n>0$.

The functions $y_{1}, y_{2}, \ldots, y_{k}$ are called the associated functions of the eigenfunction $y_{0}$ if they belong to $D(L)$ and the equalities $L y_{j}=\lambda_{0} y_{j}+y_{j-1}, j=1,2, \ldots, k$, hold.

The completeness of the system of all eigen- and associated functions of $L$ is equivalent to the completeness of the system of all root functions of this operator.

If, for the operator $L$ with dense domain $D(L)$ in $H$, the inequality $\mathfrak{J}(L y, y) \geq 0(y \in D(L))$ holds, then $L$ is called dissipative. 
Theorem 1 (see [26]). Let $L$ be an invertible operator. Then, $-L$ is dissipative if and only if the inverse operator $L^{-1}$ of $L$ is dissipative.

It is known that a kernel $A(t, s)$ of an integral operator $\mathscr{A}$ defned by

$$
\mathscr{A} f=\int_{a}^{b} A(t, s) f(s) d s, \quad f \in L^{2}(a, b),
$$

where $-\infty \leq a<b \leq \infty$, is called a Hilbert-Schmidt kernel if $|A(t, s)|^{2}$ is integrable on $(a, b)^{2}$

$$
\int_{a}^{b} \int_{a}^{b}|A(t, s)|^{2} d t d s<\infty
$$

A kernel satisfying the property $A(t, s)=\overline{A(s, t)}$ is called a Hermitian kernel. Properties of Hermitian kernels and related integral operators can be found in [27] (further see [28]). Now we shall remind some results. Let us consider the equation which is related with (1) as

$$
\mu \mathscr{A} f=f .
$$

The function $f$ which differs from zero is called a characteristic function of $\mathscr{A}$ which corresponds to the characteristic value $\mu$. If a Hermitian Hilbert-Schmidt kernel is not null then it posseses at least one characteristic value and this characteristic value (every characteristic value) is real. Further there is a finite orthonormal base of characteristic functions for each characteristic value $\mu$ of $\mathscr{A}$. Using the union of these bases one obtains an orthonormal system of characteristic functions of the kernel $A(t, s)$. If $\varphi_{1}, \varphi_{2}, \ldots, \varphi_{n}$ are distinct members of such a system belonging respectively to the characteristic value $\mu_{1}, \mu_{2}, \ldots, \mu_{n}$ (not necessarily all different) then for $1 \leq v \leq n, \varphi_{v} \otimes \varphi_{v}=\varphi_{v}(t) \overline{\varphi_{v}(s)}$ is an orthonormal system of two variables. For such a system the equivalence

$$
A(t, s) \stackrel{\circ}{=} \sum_{n=1}^{\infty} \frac{\varphi_{n} \otimes \varphi_{n}}{\mu_{n}}
$$

holds. Moreover if $g=\mathscr{A} f, f \in L^{2}$, then the equivalence

$$
g \circ \sum_{n=1}^{\infty}\left(g, \varphi_{n}\right) \varphi_{n}=\sum_{n=1}^{\infty} \frac{\left(f, \varphi_{n}\right)}{\mu_{n}} \varphi_{n}
$$

holds. Further the series in (5) are relatively uniformly absolutely convergent. However, equivalence can be replaced by equality. Before showing this, let us consider the equality

$$
g(t)=\lambda \int A(t, s) f(s) d s .
$$

Following the same idea of [27, pages 22] if $A(t, s)$ is an $L^{2}$ kernel such that $A(t, s)$ is continuous and $f$ is an $L^{2}$-function then $g(t)$ is continuous.

Hence if $A(t, s)$ is a continuous Hermitian $L^{2}$-kernel (see [27, pages 127]) and $g=\mathscr{A} f, f \in L^{2}$, then the equality

$$
g(t)=\sum_{n=1}^{\infty}\left(g, \varphi_{n}\right) \varphi_{n}(t)=\sum_{n=1}^{\infty} \frac{\left(f, \varphi_{n}\right)}{\mu_{n}} \varphi_{n}(t)
$$

holds and the series are uniformly absolutely convergent. In this case taking $g(t)=(f, \overline{A(t, \cdot)})$ the series given in (4) converge to the continuous kernel $A(t, s)$ uniformly in the variable $t$ for every $s$. Hence from (4) one can get

$$
A(t, t)=\sum_{n=1}^{\infty} \frac{\left|\varphi_{n}(t)\right|^{2}}{\mu_{n}} .
$$

Integrating both side from $a$ to $b$ we obtain

$$
\int_{a}^{b} A(t, t) d t=\sum_{n=1}^{\infty} \frac{1}{\mu_{n}} .
$$

Consequently if $A(t, t)$ is integrable on $(a, b)$ then the series converge. This implies that $\mathscr{A}$ is of trace-class (nuclear). For the definition and properties of trace-class operators see, for example, [21].

Above arguments given in [27] hold for Hermitian kernels. However from arbitrary $L^{2}$-kernels one can pass to the Hermitian kernels. For example $K_{1}(t, s)=A(t, s)+\overline{A(s, t)}$ and $K_{2}(t, s)=i(A(t, s)-\overline{A(s, t)})$ are Hermitian kernels. These Hermitian kernels are continuous and $L^{2}$-kernels if $A(t, s)$ is so. Hence above arguments hold for $K_{1}(t, s)$ and $K_{2}(t, s)$ if $A(t, s)$ is continuous $L^{2}$-kernel such that $A(t, t)$ is integrable on $(a, b)$. Further

$$
A=\frac{1}{2}\left(K_{1}-i K_{2}\right) .
$$

Hence if $K_{1}$ and $K_{2}$ are trace-class kernels then so is $A$. However this result has been given in [29, pages 526] as a definition.

Lidskil's Theorem (see [21, page 231]). If the dissipative operator $L$ is the trace class operator, then its system of root functions is complete in the Hilbert space $H$.

\section{Statement of the Problem}

The fourth order differential expression is considered as

$$
\eta(y)=y^{i v}+q(x) y, \quad x \in \Lambda:=[a, b),
$$

where $-\infty<a<b \leq \infty, a$ is the regular point and $b$ is the singular point for $\eta$, and $q$ is real-valued, Lebesgue measurable, and locally integrable function on $\Lambda$.

Let $L^{2}(\Lambda)$ be the Hilbert space consisting of all functions $y$ such that $\int_{a}^{b}|y(x)|^{2} d x<\infty$ with the inner product $(y, \chi)=$ $\int_{a}^{b} y(x) \bar{\chi}(x) d x$

Let

$\Omega=\left\{y \in L^{2}(\Lambda): y, y^{\prime}, y^{\prime \prime}, y^{\prime \prime \prime} \in \mathrm{AC}_{\mathrm{loc}}(\Lambda), \eta(y) \in L^{2}(\Lambda)\right\}$,

where $\mathrm{AC}_{\text {loc }}(\Lambda)$ denotes the set of all locally absolutely continuous functions on $\Lambda$. For arbitrary $y, \chi \in \Omega$, Green's formula is obtained as

$$
\begin{gathered}
\int_{x_{1}}^{x_{2}} \eta(y) \chi(x) d x-\int_{x_{1}}^{x_{2}} y(x) \eta(\chi) d x \\
=[y, \chi]\left(x_{2}\right)-[y, \chi]\left(x_{1}\right),
\end{gathered}
$$


where $a \leq x_{1}<x_{2} \leq b$ and $[y, \chi](x)=y^{\prime \prime \prime}(x) \chi(x)-$ $y(x) \chi^{\prime \prime \prime}(x)+y^{\prime}(x) \chi^{\prime \prime}(x)-y^{\prime \prime}(x) \chi^{\prime}(x)$. Green's formula implies the fact that if $y(x, \lambda)$ and $\chi(x, \lambda)$ both satisfy the equation $\eta(y)=\lambda y$ for the same value of $\lambda$, then $[y, \chi](x)$ is independent of $x$ and depends only on $\lambda$. Further for arbitrary $y, \chi \in \Omega$, at singular point $b$, the limits $[y, \chi](b)=$ $\lim _{x \rightarrow b}[y, \chi](x)$ and $[y, \bar{\chi}](b)=\lim _{x \rightarrow b}[y, \bar{\chi}](x)$ exist and are finite. The latter also follows from Green's formula. In fact, it is sufficient to get the second factors with their complex conjugates on the left-hand side in Green's formula.

In this paper it is assumed that $q(x)$ satisfies the lim- 4 case conditions at $b$ (see [30] and references therein). Lim-4 case is also known as Weyl's limit-circle case [8].

Let us consider the solutions $\varphi_{1}(x, \lambda), \varphi_{2}(x, \lambda), \psi_{1}(x, \lambda)$, and $\psi_{2}(x, \lambda)$ of the equation

$$
y^{i v}+q(x) y=\lambda y, \quad x \in \Lambda,
$$

where $\lambda$ is some complex parameter, satisfying the conditions

$$
\begin{aligned}
& {\left[\begin{array}{cccc}
\varphi_{1}(a, \lambda) & \varphi_{2}(a, \lambda) & \psi_{1}(a, \lambda) & \psi_{2}(a, \lambda) \\
\varphi_{1}^{\prime}(a, \lambda) & \varphi_{2}^{\prime}(a, \lambda) & \psi_{1}^{\prime}(a, \lambda) & \psi_{2}^{\prime}(a, \lambda) \\
\varphi_{1}^{\prime \prime}(a, \lambda) & \varphi_{2}^{\prime \prime}(a, \lambda) & \psi_{1}^{\prime \prime}(a, \lambda) & \psi_{2}^{\prime \prime}(a, \lambda) \\
\varphi_{1}^{\prime \prime \prime}(a, \lambda) & \varphi_{2}^{\prime \prime \prime}(a, \lambda) & \psi_{1}^{\prime \prime \prime}(a, \lambda) & \psi_{2}^{\prime \prime \prime}(a, \lambda)
\end{array}\right]} \\
& =\left[\begin{array}{cccc}
\alpha_{2} & 0 & \gamma_{1} & 0 \\
0 & \beta_{2} & 0 & \theta_{1} \\
0 & -\beta_{1} & 0 & -\theta_{2} \\
-\alpha_{1} & 0 & -\gamma_{2} & 0
\end{array}\right],
\end{aligned}
$$

where all $\alpha_{i}, \beta_{i}, \gamma_{i}$, and $\theta_{i}(i=1,2)$ are real numbers such that $\alpha_{2} \gamma_{2}-\alpha_{1} \gamma_{1}=1$ and $\beta_{1} \theta_{1}-\beta_{2} \theta_{2}=1$. For the existence of these solutions, see $[7,8,13]$. Since lim- 4 case holds for $\eta$, all solutions $\varphi_{i}(x, \lambda)$ and $\psi_{i}(x, \lambda)$ belong to $L^{2}(\Lambda)$. It is clear that $\left[\varphi_{r}, \psi_{s}\right](a)=\delta_{r s}(1 \leq r, s \leq 2)$, where $\delta_{r s}$ is the Kronocker delta, $\left[\varphi_{r}, \varphi_{s}\right](a)=0$ and $\left[\psi_{r}, \psi_{s}\right](a)=0$.

Let us set $u_{i}(x)=\varphi_{i}(x, 0)$ and $z_{i}(x)=\psi_{i}(x, 0)(i=$ $1,2)$, with $x \in \Lambda$. Then $u_{i}$ and $z_{i}$ become the real solutions of $\eta(y)=0(x \in \Lambda)$. Further, they belong to $\Omega$. Hence for arbitrary $y \in \Omega$, the values $\left[y, u_{1}\right](b),\left[y, u_{2}\right](b),\left[y, z_{1}\right](b)$, and $\left[y, z_{2}\right](b)$ exist and are finite.

Let $D(N)$ be the set of all functions $y \in \Omega$ satisfying the boundary conditions

$$
\begin{gathered}
\alpha_{1} y(a)+\alpha_{2} y^{\prime \prime \prime}(a)=0, \\
\beta_{1} y^{\prime}(a)+\beta_{2} y^{\prime \prime}(a)=0, \\
{\left[y, u_{1}\right](b)-h_{1}\left[y, z_{1}\right](b)=0,} \\
{\left[y, u_{2}\right](b)-h_{2}\left[y, z_{2}\right](b)=0,}
\end{gathered}
$$

where $\alpha_{i}$ and $\beta_{i}(i=1,2)$ are real numbers given as previously stated and $h_{1}$ and $h_{2}$ are some complex numbers such that $h_{1}=\mathfrak{R} h_{1}+i \Im h_{1}$ and $h_{2}=\mathfrak{R} h_{2}+i \Im h_{2}$ with $\Im h_{i}>0, i=1,2$.

It should be noted that for any solutions $y(x, \lambda)$ of $(14)$, conditions (16) and (17), respectively, can be written as

$$
\begin{aligned}
& {\left[y, \varphi_{1}\right](a)=0,} \\
& {\left[y, \varphi_{2}\right](a)=0 .}
\end{aligned}
$$

The operator $N$ is defined on $D(N)$ as $N y=\eta(y), y \in$ $D(N), x \in \Lambda$. The main aim of this paper is to investigate the spectral analysis of the operator $N$ (the boundary value problem (14)-(19)).

\section{Completeness Theorems}

Let $\left\{y_{j}(x, \lambda) ; 1 \leq j \leq r\right\}$ be any $r(1 \leq r \leq 4)$ solutions of $\eta(y)=\lambda y$. The notation $W\left\{y_{1}, y_{2}, \ldots, y_{r}\right\}=W\left\{y_{1}(x, \lambda)\right.$, $\left.y_{2}(x, \lambda), \ldots, y_{r}(x, \lambda)\right\}$ denotes the Wronskian of order $r$ of this set of functions $[7,8,31]$.

It is known that the following equality holds $[7,8,13]$ :

$$
\begin{aligned}
W\left\{y_{1}, y_{2}, y_{3}, y_{4}\right\}= & -\left[y_{1}, y_{2}\right](x)\left[y_{3}, y_{4}\right](x) \\
& +\left[y_{1}, y_{3}\right](x)\left[y_{2}, y_{4}\right](x) \\
& -\left[y_{1}, y_{4}\right](x)\left[y_{2}, y_{3}\right](x)
\end{aligned}
$$

This equation also shows that $W\left\{y_{1}, y_{2}, y_{3}, y_{4}\right\}$ of any four solutions of $\eta(y)=\lambda y$ is independent of $x$ and depends only on $\lambda[7,8]$.

Now consider the solutions $\vartheta_{1}(x, \lambda)$ and $\vartheta_{2}(x, \lambda)$ of the equation $\eta(y)=\lambda y, x \in \Lambda$, satisfying the conditions

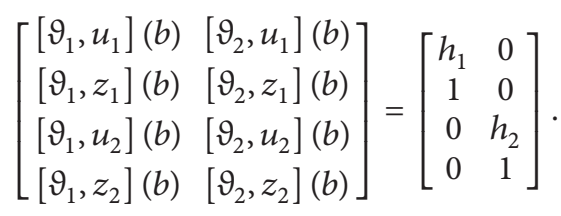

For the existence of these solutions given with the previous conditions, see [13]. Clearly these solutions satisfy conditions (18) and (19), respectively. Now let us set

$$
W(\lambda)=W\left\{\varphi_{1}, \varphi_{2}, \vartheta_{1}, \vartheta_{2}\right\}
$$

Then $W(\lambda)$ becomes an entire function, and the zeros of $W(\lambda)$ coincide with the eigenvalues of the operator $N$ [13]. This implies that all zeros of $W$ (all eigenvalues of $N$ ) are discrete and that possible limit points of these zeros (eigenvalues of $N)$ can only occur at infinity.

Using (22), we immediately have

$$
\begin{aligned}
& W\left\{u_{1}, u_{2}, z_{1}, z_{2}\right\}=1, \\
& {\left[\begin{array}{llll}
{\left[u_{1}, u_{1}\right](x)} & {\left[u_{2}, u_{1}\right](x)} & {\left[z_{1}, u_{1}\right](x)} & {\left[z_{2}, u_{1}\right](x)} \\
{\left[u_{1}, u_{2}\right](x)} & {\left[u_{2}, u_{2}\right](x)} & {\left[z_{1}, u_{2}\right](x)} & {\left[z_{2}, u_{2}\right](x)} \\
{\left[u_{1}, z_{1}\right](x)} & {\left[u_{2}, z_{1}\right](x)} & {\left[z_{1}, z_{1}\right](x)} & {\left[z_{2}, z_{1}\right](x)} \\
{\left[u_{1}, z_{2}\right](x)} & {\left[u_{2}, z_{2}\right](x)} & {\left[z_{1}, z_{2}\right](x)} & {\left[z_{2}, z_{2}\right](x)}
\end{array}\right]} \\
& =\left[\begin{array}{cccc}
0 & 0 & -1 & 0 \\
0 & 0 & 0 & -1 \\
1 & 0 & 0 & 0 \\
0 & 1 & 0 & 0
\end{array}\right] .
\end{aligned}
$$


Hence, the Plücker identity for the fourth-order case is obtained (see [13, p. 435]):

$$
\begin{aligned}
{[y, \chi](x)=} & {\left[y, u_{1}\right](x)\left[\chi, z_{1}\right](x) } \\
& -\left[y, z_{1}\right](x)\left[\chi, u_{1}\right](x) \\
& +\left[y, u_{2}\right](x)\left[\chi, z_{2}\right](x) \\
& -\left[y, z_{2}\right](x)\left[\chi, u_{2}\right](x),
\end{aligned}
$$

where $y, \chi \in D(N)$.

Theorem 2. The operator $N$ is dissipative in $L^{2}(\Lambda)$.

Proof. For $y \in D(N)$, we have

$$
(N y, y)-(y, N y)=[y, \bar{y}](b)-[y, \bar{y}](a) .
$$

Since $y \in D(N)$, a direct calculation shows that

$$
[y, \bar{y}](a)=0 \text {. }
$$

Further, using (26) and conditions (18) and (19) one obtains

$$
[y, \bar{y}](b)=2 i \Im h_{1}\left|\left[y, z_{1}\right](b)\right|^{2}+2 i \Im h_{2}\left|\left[y, z_{2}\right](b)\right|^{2} .
$$

Substituting (28) and (29) into (27) it is obtained that

$$
\mathfrak{I}(N y, y)=\mathfrak{\Im} h_{1}\left|\left[y, z_{1}\right](b)\right|^{2}+\mathfrak{\Im} h_{2}\left|\left[y, z_{2}\right](b)\right|^{2},
$$

and this completes the proof.

Theorem 2 shows that all eigenvalues of $N$ lie in the closed upper half-plane.

Theorem 3. The operator $N$ has no real eigenvalue.

Proof. For $y \in D(N)$, a direct calculation shows that

$$
\mathfrak{I}(N y, y)=\mathfrak{I}\left(\lambda\|y\|^{2}\right) .
$$

Now let $\lambda=\lambda_{0}$ be a real eigenvalue of $N$, and let $\varphi_{1}\left(x, \lambda_{0}\right)$ be the corresponding eigenfunction. Then (31) and (30) gives $\left[\varphi_{1}, z_{1}\right](b)=\left[\varphi_{1}, z_{2}\right](b)=0$. Using these equalities in (18) and (19) one gets that $\left[\varphi_{1}, u_{1}\right](b)=\left[\varphi_{1}, u_{2}\right](b)=0$.

Let us consider the solution $\psi_{1}\left(x, \lambda_{0}\right)$. Hence, using (26) it is obtained that

$$
\begin{aligned}
1= & {\left[\varphi_{1}, \psi_{1}\right](a)=\left[\varphi_{1}, \psi_{1}\right](b) } \\
= & {\left[\varphi_{1}, u_{1}\right](b)\left[\psi_{1}, z_{1}\right](b) } \\
& -\left[\varphi_{1}, z_{1}\right](b)\left[\psi_{1}, u_{1}\right](b) \\
& +\left[\varphi_{1}, u_{2}\right](b)\left[\psi_{1}, z_{2}\right](b) \\
& -\left[\varphi_{1}, z_{2}\right](b)\left[\psi_{1}, u_{2}\right](b)=0 .
\end{aligned}
$$

This contradiction completes the proof.

From Theorem 3 it is obtained that all eigenvalues of $N$ lie in the open upper half-plane. In particular zero is not an eigenvalue of $N$. Hence, the operator $N^{-1}$ exists.
Consider the solutions $u_{1}(x), u_{2}(x), v_{1}(x)$ and $v_{2}(x)$, where $v_{1}(x)=u_{1}(x)-h_{1} z_{1}(x)$ and $v_{2}(x)=u_{2}(x)-$ $h_{2} z_{2}(x) . u_{1}(x)$ and $u_{2}(x)$ satisfy conditions (16) and (17), respectively and $v_{1}(x)$ and $v_{2}(x)$ satisfy conditions (18) and (19), respectively.

The equation $N y=f(x), y \in D(N), x \in \Lambda$, is equivalent to the nonhomogeneous differential equation

$$
y^{i v}+q(x) y=f(x), \quad y \in D(N), x \in \Lambda,
$$

subject to the boundary conditions

$$
\begin{gathered}
{\left[y, u_{1}\right](a)=0,} \\
{\left[y, u_{2}\right](a)=0,} \\
{\left[y, u_{1}\right](b)-h_{1}\left[y, z_{1}\right](b)=0,} \\
{\left[y, u_{2}\right](b)-h_{2}\left[y, z_{2}\right](b)=0}
\end{gathered}
$$

(compare the boundary conditions at $a$ with (20) and (21)). Using Everitt's method [7] (further see [8, 13]) the general solution is obtained as

$$
y(x)=\int_{a}^{b} G(x, \zeta) f(\zeta) d \zeta,
$$

where

$$
G(x, \zeta)= \begin{cases}v^{T}(\zeta) u(x), & a \leq x<\zeta, \\ v^{T}(x) u(\zeta), & \zeta<x \leq b,\end{cases}
$$

$v(x)=u(x)-h z(x)$ and

$$
u(x)=\left(\begin{array}{l}
u_{1}(x) \\
u_{2}(x)
\end{array}\right), \quad z(x)=\left(\begin{array}{l}
z_{1}(x) \\
z_{2}(x)
\end{array}\right), \quad h=\left(\begin{array}{cc}
h_{1} & 0 \\
0 & h_{2}
\end{array}\right) .
$$

The operator $K$ defined by

$$
K f=\int_{a}^{b} G(x, \zeta) f(\zeta) d \zeta
$$

where $f \in L^{2}(\Lambda)$, is the inverse operator of $N$. Hence the completeness of the system of all eigen- and associated functions of $N$ is equivalent to the completeness of those of $K$ in $L^{2}(\Lambda)$.

Since $G(x, \zeta)$ is a continuous Hilbert-Schmidt kernel and $G(x, x)$ is integrable on $[a, b)$, the operator $K$ is of trace class.

Let us consider the operator $-K$. Since $N$ is dissipative in $L^{2}(\Lambda),-K$ is also dissipative in $L^{2}(\Lambda)$. Thus all conditions are satisfied for Lidskiı̌'s theorem. So we have the following.

Theorem 4. The system of all root functions of $-K$ (also $K$ ) is complete in $L^{2}(\Lambda)$.

Since the completeness of the system of root functions (eigen- and associated functions) of $K$ is equivalent to the completeness of those of $N$, is obtained that the following.

Theorem 5. All eigenvalues of the problem (14)-(19) lie in the open upper half-plane and they are purely discrete. The system of all eigen- and associated functions of the problem (14)-(19) is complete in $L^{2}(\Lambda)$. 


\section{References}

[1] H. Weyl, "Über gewöhnliche Differentialgleichungen mit Singularitäten und die zugehörigen Entwicklungen willkürlicher Funktionen," Mathematische Annalen, vol. 68, no. 2, pp. $220-$ 269, 1910 .

[2] E. C. Titchmarsh, Eigenfunction Expansions Associated with Second Order Differential Equations, Part 1, Oxford University Press, 2nd edition, 1962.

[3] E. A. Coddington and N. Levinson, Theory of Ordinary Differential Equations, McGraw-Hill, New York, NY, USA, 1955.

[4] M. A. Naimark, Linear Differential Operators, Nauka, Moscow, Russia, 2nd edition, 1969, English translation, Ungar, New York, NY, USA, 1st edition, parts 1, 1967, 1st edition, parts 2, 1968.

[5] I. M. Glazman, Direct Methods of Qualitative Spectral Analysis of Singular Differential Operators, Israel Program for Scientific Translations, Jerusalem, Israel, 1965.

[6] F. V. Atkinson, Discrete and Continuous Boundary Problems, Academic Press, New York, NY, USA, 1964.

[7] W. N. Everitt, "The Sturm-Liouville problem for fourth-order differential equations," The Quarterly Journal of Mathematics, vol. 8, pp. 146-160, 1957.

[8] W. N. Everitt, "Fourth order singular differential equations," Mathematische Annalen, vol. 149, pp. 320-340, 1963.

[9] W. N. Everitt, "Singular differential equations. I. The even order case," Mathematische Annalen, vol. 156, pp. 9-24, 1964.

[10] W. N. Everitt, "Singular differential equations. II. Some selfadjoint even order cases," The Quarterly Journal of Mathematics, vol. 18, pp. 13-32, 1967.

[11] W. N. Everitt, D. B. Hinton, and J. S. W. Wong, "On the strong limit- $n$ classification of linear ordinary differential expressions of order $2 n$," Proceedings of the London Mathematical Society, vol. 29, pp. 351-367, 1974.

[12] V. I. Kogan and F. S. Rofe-Beketov, "On square-integrable solutions of symmetric systems of differential equations of arbitrary order," Proceedings of the Royal Society of Edinburgh A, vol. 74, pp. 5-40, 1974.

[13] C. T. Fulton, "The Bessel-squared equation in the lim-2, lim-3 and lim-4 cases," The Quarterly Journal of Mathematics, vol. 40, no. 160 , pp. 423-456, 1989.

[14] A. M. Krall, " $M(\lambda)$ theory for singular Hamiltonian systems with one singular point," SIAM Journal on Mathematical Analysis, vol. 20, no. 3, pp. 664-700, 1989.

[15] A. M. Krall, " $M(\lambda)$ theory for singular Hamiltonian systems with two singular points," SIAM Journal on Mathematical Analysis, vol. 20, no. 3, pp. 700-715, 1989.

[16] D. B. Hinton and J. K. Shaw, "On Titchmarsh-Weyl $M(\lambda)$-functions for linear Hamiltonian systems," Journal of Differential Equations, vol. 40, no. 3, pp. 316-342, 1981.

[17] D. B. Hinton and J. K. Shaw, "Hamiltonian systems of limit point or limit circle type with both endpoints singular," Journal of Differential Equations, vol. 50, no. 3, pp. 444-464, 1983.

[18] D. B. Hinton and J. K. Shaw, "Parameterization of the $M(\lambda)$ function for a Hamiltonian system of limit circle type," Proceedings of the Royal Society of Edinburgh A, vol. 93, no. 3-4, pp. 349360,1983 .

[19] D. B. Hinton and J. K. Shaw, "On boundary value problems for Hamiltonian systems with two singular points," SIAM Journal on Mathematical Analysis, vol. 15, no. 2, pp. 272-286, 1984.
[20] P. W. Walker, "A vector-matrix formulation for formally symmetric ordinary differential equations with applications to solutions of integrable square," Journal of the London Mathematical Society, vol. 9, no. 2, pp. 151-159, 1974/75.

[21] I. C. Gohberg and M. G. Kreĭn, Introduction to the Theory of Linear Nonselfadjoint Operators, American Mathematical Society, Providence, RI, USA, 1969.

[22] B. S. Pavlov, "Spectral analysis of a dissipative singular Schrödinger operator in terms of a functional model," in Partial Differential Equations, vol. 65 of Itogi Nauki i Tekhniki. Seriya Sovremennye Problemy Matematiki. Fundamental'nye Napravleniya, pp. 95-163, 1991, English translation in Partial Differential Equations VIII, vol. 65 of Encyclopaedia of Mathematical Sciences, pp. 87-163, 1996.

[23] G. Guseinov, "Completeness theorem for the dissipative SturmLiouville operator," Doga. Turkish Journal of Mathematics, vol. 17, no. 1, pp. 48-54, 1993.

[24] E. Bairamov and A. M. Krall, "Dissipative operators generated by the Sturm-Liouville differential expression in the Weyl limit circle case," Journal of Mathematical Analysis and Applications, vol. 254, no. 1, pp. 178-190, 2001.

[25] E. Uğurlu and E. Bairamov, "Dissipative operators with impulsive conditions," Journal of Mathematical Chemistry, vol. 51, pp. $1670-1680,2013$.

[26] Z. Wang and H. Wu, "Dissipative non-self-adjoint SturmLiouville operators and completeness of their eigenfunctions," Journal of Mathematical Analysis and Applications, vol. 394, no. 1, pp. 1-12, 2012.

[27] F. Smithies, Integral Equations, Cambridge University Press, New York, NY, USA, 1958.

[28] F. Riesz and B. Sz-Nagy, Functional Analysis, Frederick Ungar, New York, NY, USA, 6th edition, 1972.

[29] E. Prugovečki, Quantum Mechanics in Hilbert Space,, vol. 92, Academic Press, New York, NY, USA, 2nd edition, 1981.

[30] M. S. P. Eastham, “The limit- $2 n$ case of symmetric differential operators of order $2 n$," Proceedings of the London Mathematical Society, vol. 38, no. 2, pp. 272-294, 1979.

[31] E. L. Ince, Ordinary Differential Equations, London, UK, 1927. 


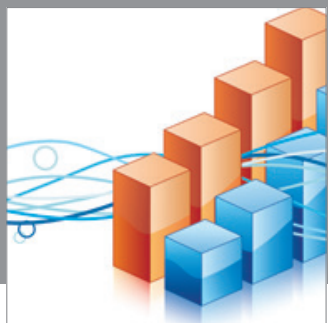

Advances in

Operations Research

mansans

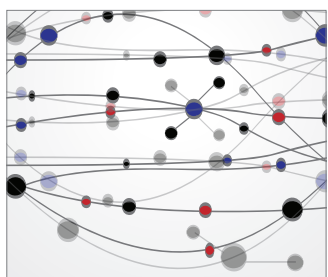

The Scientific World Journal
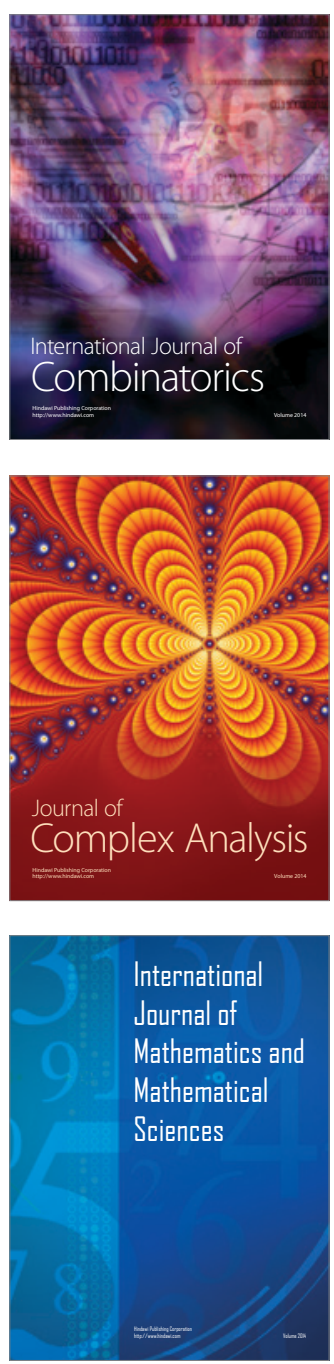
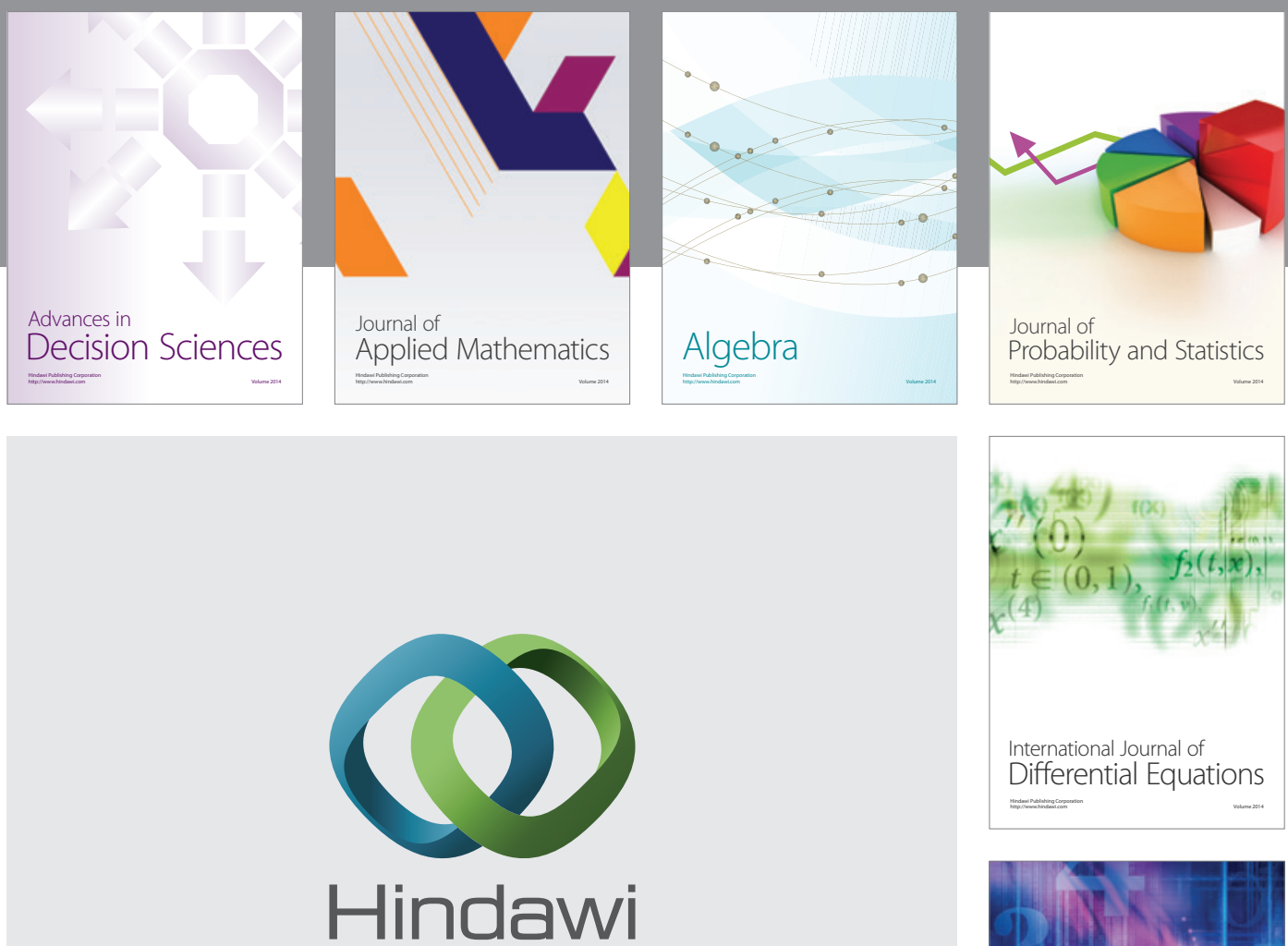

Submit your manuscripts at http://www.hindawi.com
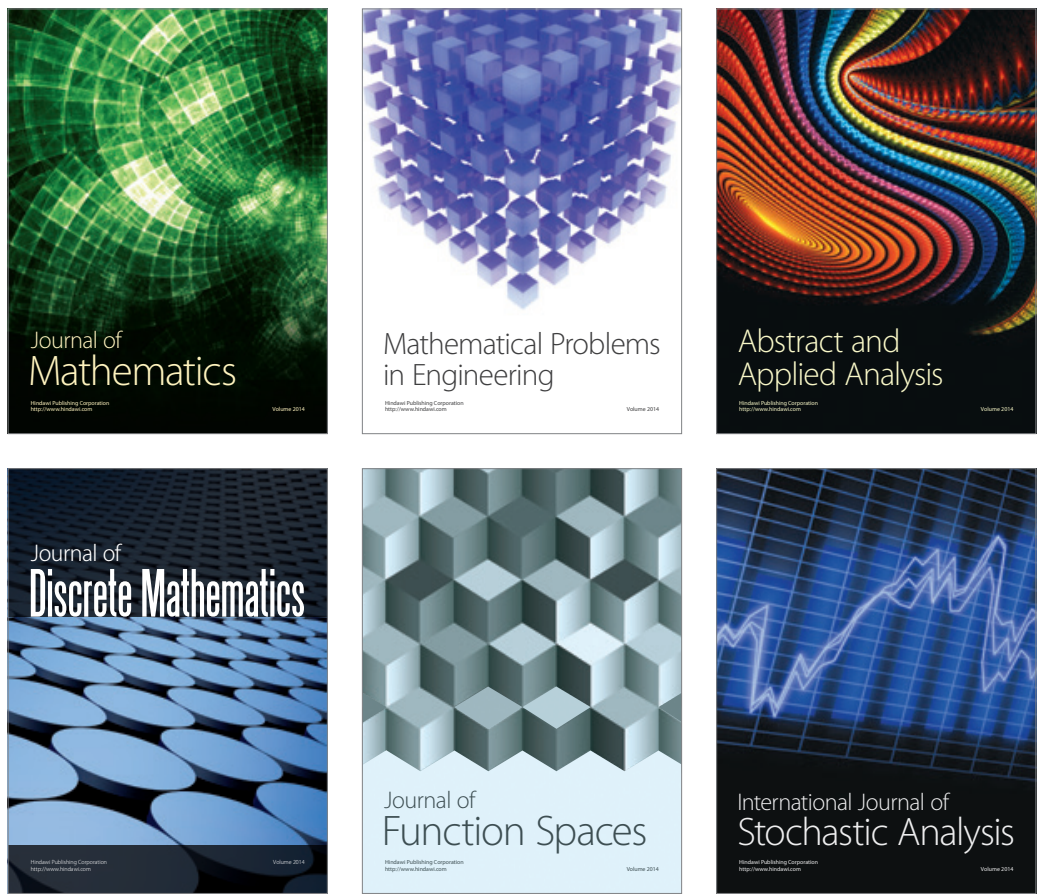

Journal of

Function Spaces

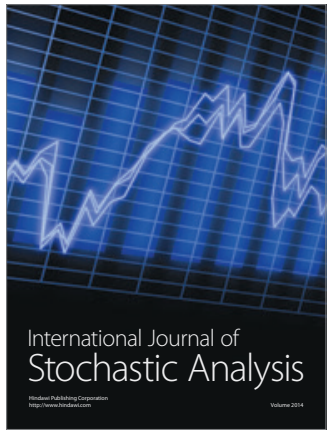

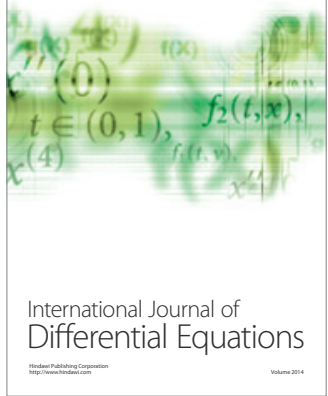
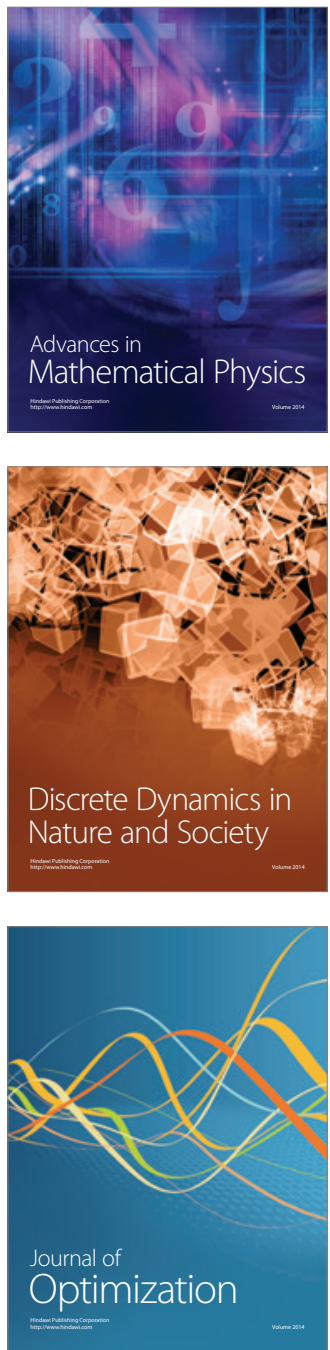